\title{
Mapping Diversity of Publication Patterns in the Social Sciences and Humanities: An Approach Making Use of Fuzzy Cluster Analysis
}

\author{
Frederik T. Verleysen ${ }^{1 \dagger} \&$ Arie Weeren ${ }^{2}$ \\ ${ }^{1}$ Centre for Research \& Development Monitoring (ECOOM), Faculty of Social \\ Sciences, University of Antwerp, Middelheimlaan 1, 2020 Antwerp, Belgium \\ ${ }^{2}$ Mathworks, Postbus 7921, 5605 SH Eindhoven, The Netherlands
}

Citation: Frederik T. Verleysen \& Arie Weeren (2016). Mapping Diversity of Publication Patterns in the Social Sciences and Humanities: An Approach Making Use of Fuzzy Cluster Analysis.

Received: Aug. 2, 2016 Revised: Aug. 20, 2016

Accepted: Aug. 24, 2016

Purpose: To present a method for systematically mapping diversity of publication patterns at the author level in the social sciences and humanities in terms of publication type, publication language and co-authorship.

Design/methodology/approach: In a follow-up to the hard partitioning clustering by Verleysen and Weeren in 2016, we now propose the complementary use of fuzzy cluster analysis, making use of a membership coefficient to study gradual differences between publication styles among authors within a scholarly discipline. The analysis of the probability density function of the membership coefficient allows to assess the distribution of publication styles within and between disciplines.

Findings: As an illustration we analyze 1,828 productive authors affiliated in Flanders, Belgium. Whereas a hard partitioning previously identified two broad publication styles, an international one vs. a domestic one, fuzzy analysis now shows gradual differences among authors. Internal diversity also varies across disciplines and can be explained by researchers' specialization and dissemination strategies.

Research limitations: The dataset used is limited to one country for the years 2000-2011; a cognitive classification of authors may yield a different result from the affiliation-based classification used here.

Practical implications: Our method is applicable to other bibliometric and research evaluation contexts, especially for the social sciences and humanities in non-Anglophone countries.

Originality/value: The method proposed is a novel application of cluster analysis to the field of bibliometrics. Applied to publication patterns at the author level in the social sciences and humanities, for the first time it systematically documents intra-disciplinary diversity.

\footnotetext{
Corresponding author: Frederik T. Verleysen (E-mail: Frederik.Verleysen@uantwerpen.be).
}

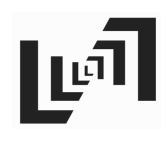

JDIS Journal of Data and Information Science

Vol. 1 No. 4, 2016 pp 33-59

DOI: $10.20309 /$ jdis. 201624 http://www.jdis.org 


\section{Research Paper}

Keywords Bibliometrics; Social sciences and humanities; Publication patterns; Dissemination; Cluster analysis

\section{Introduction}

It is a well-known fact that publication patterns in the social sciences and humanities (SSH) differ considerably from those observed in scientific, technical, and biomedical fields. SSH scholars publish their research in a much wider array of both international and domestic publication channels, including monographs and edited books; they frequently opt for other publication languages besides English; and their rate of research collaboration and ensuing co-authorship is considerably lower (Hicks, 2004; Nederhof, 2006). In recent years, bibliometric studies of the $\mathrm{SSH}$ have started to devote more attention to the topic of internal diversity. This has mostly been demonstrated by analyses at the disciplinary level, showing that an inter-disciplinary variety in terms of publication patterns exists across the spectrum of the SSH. One particular pattern described is that of a divide between most disciplines belonging to the social sciences and those classified as humanities. In the social sciences the use of international journals, English as a publication language and more frequent co-authorship are appearing to become predominant, while by contrast in the humanities books and chapters and the use of national or regional languages retain a central position, and co-authorship occurs less frequently (Engels, Ossenblok, \& Spruyt, 2012; Ossenblok, 2016; Puuska, 2014; Sivertsen, 2009).

By contrast, the intra-disciplinary diversity of publication patterns, the variety within disciplines belonging to the $\mathrm{SSH}$, has not received as much attention. A handful of recent studies have documented such diversity in terms of publication and citation patterns (Chi, 2015; Nederhof, 2011) or in that of cognitive structure, mostly of a single discipline (Lin \& Kaid, 2000; Persson, 2015). In an effort to document intra-disciplinary diversity in a more systematic way, Verleysen and Weeren (2016) have performed a hard partitioning cluster analysis on the publication patterns of 1,828 individual authors belonging to $16 \mathrm{SSH}$ disciplines and affiliated with the five universities in Flanders, Belgium. This analysis at the author level has demonstrated that intra-disciplinary diversity as regards publication patterns in Flanders is considerable, as well as that it is too simplistic to oppose the publication cultures of the social sciences to those in the humanities. For SSH scholars in Flanders two broad publication styles were identified: the first one is centered around co-authored English-language journal articles in high-profile outlets indexed by the Web of Science; the second one is far more reliant on single-authored articles

Journal of Data and in national journals and books in other languages than English, especially Dutch, the dominant language in Flanders (Verleysen \& Weeren, 2016). 
Mapping Diversity of Publication Patterns in the Social Sciences and Humanities:

An Approach Making Use of Fuzzy Cluster Analysis

Frederik T. Verleysen \& Arie Weeren

In the present article, we refine our previous results and propose additional steps

for a method for the study of diversity of publication patterns in the social sciences and humanities.

\section{Data and Method}

This paper builds upon the data, method, and results of the cluster analysis by Verleysen and Weeren (2016) of the 1,828 most productive scholarly authors (ten or more weighted peer reviewed outputs during 2000-2011) registered in the Flemish Bibliographic Database for the Social Sciences and Humanities (or VABB-SHW).

The VABB-SHW is a comprehensive regional bibliographic database (i.e. not a citation index) used for calculating a share of the research funding provided by the government to the five Flemish universities. In this capacity the VABB-SHW registers five publication types: journal articles, monographs, edited books, book chapters, and proceedings papers. For inclusion in the Flemish funding model, a weight is attributed to each publication type: journal articles, edited books and book chapters all receive a weight of 1 , whereas monographs have a weight of 4 and proceedings papers one of 0.5 . Two parts comprise the VABB-SHW. The first, VABB-WoS, consists of records of publications (journal articles and proceedings papers) which are also indexed in a journal and/or proceedings index of the Web of Science (WoS). VABB-WoS consists of ca. 95\% of English language publications, and concentrates most of the high-profile international journals in the SSH. The second part, VABB-GP, consists of records of publications which have additionally been identified as peer reviewed by the Authoritative Panel (Gezaghebbend Panel or GP), an independent scientific board of university professors, from the whole of the five universities' non-WoS publications. VABB-GP consists for ca. $70 \%$ of publications in other languages than English, especially Dutch (Engels, Ossenblok \& Spruyt, 2012).

As input for the hard partitioning cluster analysis (Verleysen \& Weeren, 2016), a dataset was compiled listing the 1,828 author names, their main disciplinary affiliation, as well as 11 variables mapping author output during 2000-2011. These variables belong to three groups of attributes which are known to differentiate SSH publication patterns at the disciplinary level: publication type, publication language, and the share of co-authored publications. For the three VABB-SHW book publication types, combined with two publication language groups (English $v s$. other languages), this resulted in a subtotal of six variables, for each of which the fractional contribution to individual authors' total 12-year weighted output was calculated. For journal articles and proceedings papers, fractions were calculated based on the distinction between VABB-WoS and VABB-GP, resulting in a subtotal of four additional variables. The $11^{\text {th }}$ variable is the fraction of weighted co-authored publications.

Journal of Data and Information Science http://www.jdis.org 


\section{Research Paper}

Mahalanobis distance or 'generalized squared interpoint distance' (Mahalanobis, 1936; Wicklin, 2015) was used to calculate dissimilarities between all possible pairs of the 1,828 authors. By means of silhouettes and the $k$-medoids clustering algorithm (Kaufman \& Rousseeuw, 1990), we arrived at a 'hard' or 'crisp' clustering result of two distinct clusters of publication patterns within the Flemish publication data. As $k$-medoids identifies the most representative object in every cluster, i.e. the object for which the distance to every other object within its cluster is minimal, a simple retrieval of the original input data for both medoids (i.e. the most representative authors) allowed to label the two clusters - that is: to describe in qualitative terms their underlying publication pattern. We were thus able to identify two distinct styles for publishing research results in Flemish SSH, both of which were found to be present in all 16 disciplines, thereby cutting across the distinction between the social sciences and the humanities (cf. Sections 1,3). For further elaboration on the method used for hard partitioning we refer to Verleysen and Weeren (2016) and Kaufman and Rousseeuw (1990).

For the present paper we take the analysis a step further by performing a fuzzy cluster analysis on the prior two-cluster result. Whereas the initial hard partitioning attributes all cases to just one of the (here: two) clusters, fuzzy clustering allows for some ambiguity in the data by calculating for each case a membership coefficient, or the degree of belonging of individual authors to each of both clusters (Kaufman $\&$ Rousseeuw, 1990). By including this additional information, binary decisions on cluster membership are avoided and the resulting picture of scholarly publication patterns should be more nuanced than the initial result. The fuzzy clustering algorithm used is Fanny by Kaufman and Rousseeuw (1990), implemented in MATLAB ${ }^{\circledR}$ R2016a. After applying the fuzzy principle to the two-cluster result by Verleysen and Weeren (2016), we present cluster plots for each individual discipline to illustrate diversity in publication patterns within and across disciplines. In a final step, histograms are used to illustrate the distribution of publication styles for each discipline, by showing the probability density function (Johnson \& Wichern, 1992), i.e. a scaling of the original author frequencies denoting the relative probability of a cluster membership coefficient value for a random author in a given discipline. As the disciplines in our analysis are different in size, recalculating absolute author frequencies into the probability density function allows for easier comparison between disciplines.

\section{Results}

Journal of Data and Information Science

Figure 1 presents the fuzzy principle applied to the two-cluster result on the 1,828 authors of Verleysen and Weeren (2016) (one author = one dot). The two cluster 
cores identified by the previous hard partitioning are still easily visible in the new result: Cluster One (bright red) and Cluster Two (bright green). By identification of the respective most representative authors (medoids) for both clusters, we previously determined (Verleysen \& Weeren, 2016) that the core of Cluster One 'groups those authors who mainly target an international audience of specialized academia, through the collaborative publication of mainly English language articles in highprofile journals indexed by the WoS'. Inversely, the core of Cluster Two contains 'those authors who are more strongly oriented towards national journals and also book publications, make frequent use of other languages than English (i.e. mainly Dutch), and are much less inclined to co-author publications (Verleysen \& Weeren, 2016).

In Figure 1, the result of the fuzzy algorithm Fanny, the belonging of individual authors to the two clusters is now visualized by various shades of red and green. This degree of fuzziness of the result is also expressed by the normalized version of Dunn's partition coefficient (Dunn, 1976), which on a 0-1 scale gives an indication of how hard or fuzzy the clustering result is. A value of 0 would denote that each object (author) has equal membership in each cluster, or that the result is entirely fuzzy; a value of 1 would mean that each object has a membership of 1 in one cluster and a membership of 0 in the other cluster, or that the result is entirely hard. For the clustering of the 1,828 authors by means of the Fanny algorithm, Dunn's normalized partition coefficient has a value of 0.2390 , demonstrating the appropriateness of the fuzzy approach.

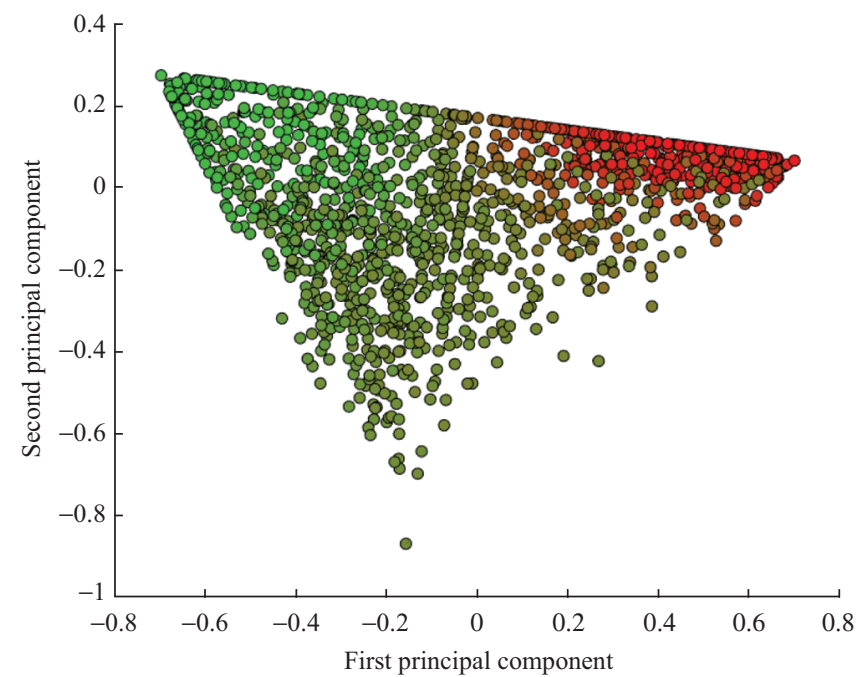

Figure 1. Fuzzy clustering of 1,828 productive authors in the SSH $(n=1,828)$.

Journal of Data and Information Science http://www.jdis.org 


\section{Research Paper}

The second part of this Section presents the clustering plots for authors belonging to two examples of individual SSH disciplines, Sociology (social sciences) and Linguistics (humanities). Plots and histograms for all other disciplines can be found in the Appendix. The histograms show the probability density function (y-axis) for the cluster membership coefficient of all authors affiliated with a discipline on a 0-1 scale ( $x$-axis), whereby a value of 0 of the coefficient denotes a $100 \%$ membership of Cluster Two (green) and a value of 1 a 100\% membership of Cluster One (red). For the comparison between disciplines of the histograms we note the existence of a scale variation on the $y$-axis (probability density function). This results from the considerable variety between disciplines regarding the concentration of cluster membership coefficient values, as the more strongly concentrated within certain ranges of the coefficient the outcomes for individual authors are, the higher the probability of a random author's value occurring within this same range. As in this paper we focus primarily on intra-disciplinary diversity, for which maximum legibility of the individual histograms is required, we opted not to standardize the scale of the probability density function. On the $x$-axis (membership coefficient), the bin width of the histograms is the software default calculated by the varying number of cases in each discipline, mentioned in the figure legends.

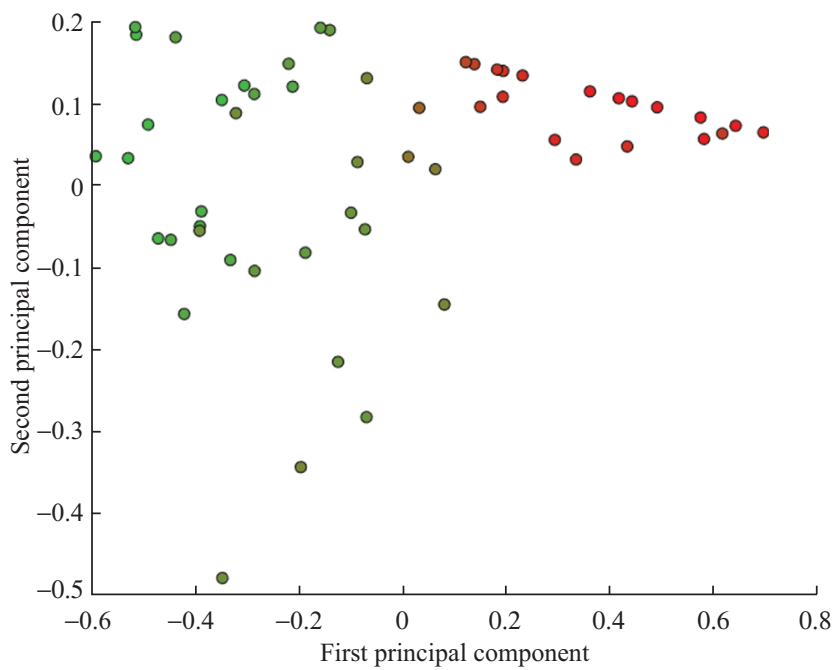

Figure 2. Fuzzy clustering of productive authors in Sociology $(n=57)$.

For both Sociology and Linguistics, the cluster plots and histograms (Figures 2-5)

Journal of Data and show that intra-disciplinary diversity of publication patterns occurs across a wide Information Science spectrum. While linguists show by far the strongest presence on the fringes of 


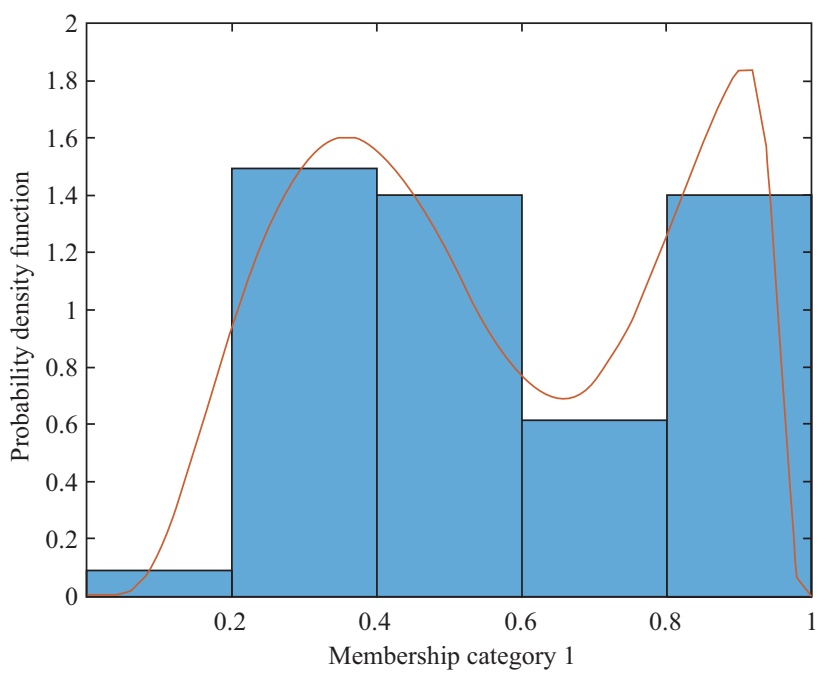

Figure 3. Probability density function of cluster membership coefficients for Sociology $(n=57)$.

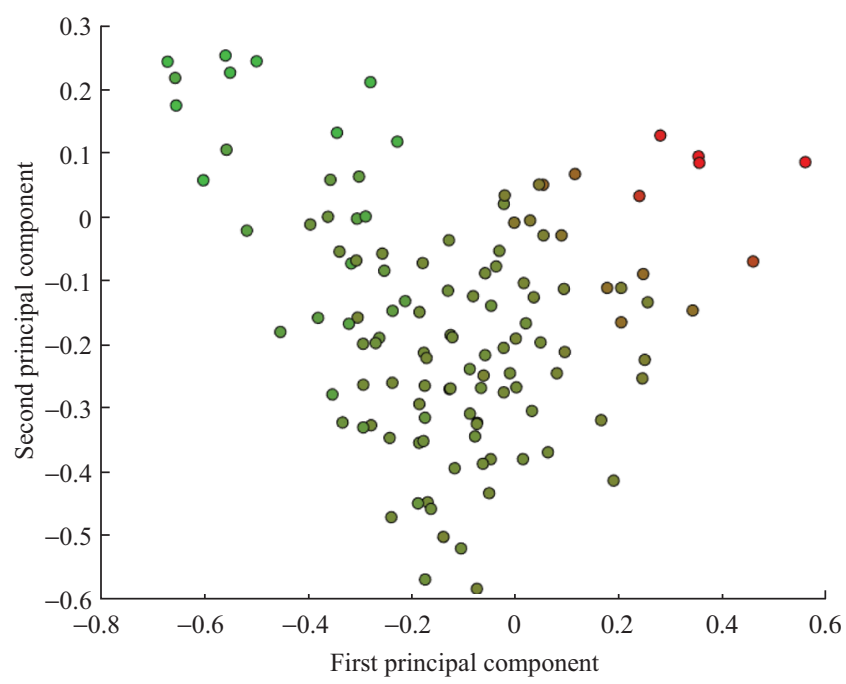

Figure 4. Fuzzy clustering of productive authors in Linguistics $(n=121)$.

Cluster Two (dark green), a limited number of them clearly belong to Cluster One (red), with an equally modest number of authors (brown) occupying the middle ground of Cluster One. In the histogram this predominance of Cluster Two is confirmed by the value of the probability density function for the membership coefficient range of $0.4-0.5$, which, though near the center between both clusters, 


\section{Research Paper}

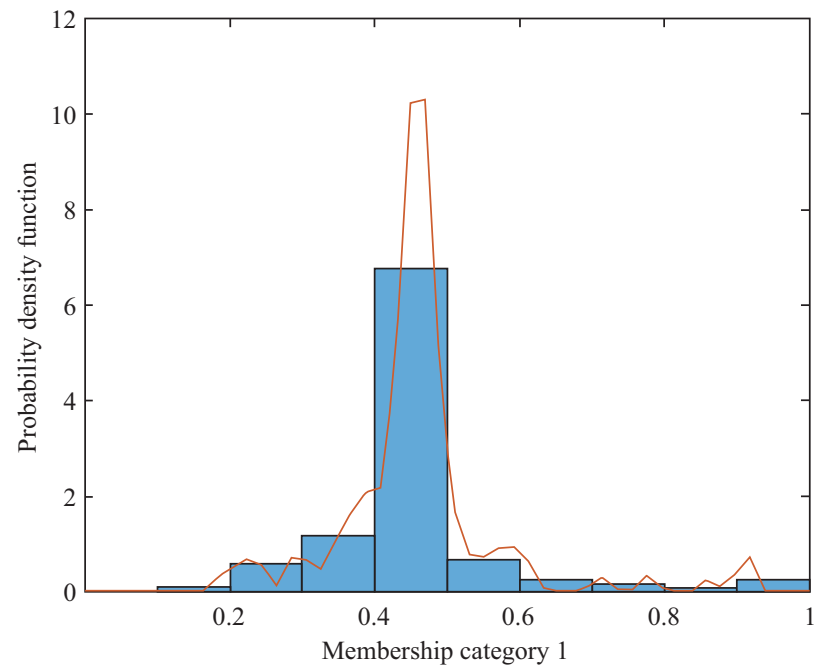

Figure 5. Probability density function of cluster membership coefficients for Linguistics $(n=121)$.

is still closer to that of Cluster Two. For sociologists the divide between publication patterns within the discipline is more profound. Bright green and bright red dots (authors) are dominant, with relatively fewer authors occupying the middle ground (dark green and brown). The probability density function confirms this outspoken divide between publication styles within the discipline.

\section{Discussion}

Cluster analysis based on bibliographic data for individual researchers reveals how publication patterns can differ widely between authors affiliated with the same discipline. It also demonstrates how publication patterns of social scientists cannot simplistically be opposed to those of humanities scholars. At the same time, there remain considerable differences between the Flemish SSH disciplines used as an example here. Several of the humanities such as Art History, History, Law, Literature, and Theology show a concentration of researchers who publish most often in national journals and books, make use of other languages besides English, and who frequently publish on their own. Other humanities such as Archeology, Communication Studies, Linguistics, and Philosophy show a more dispersed pattern, with a number of their researchers clearly adhering to the other publication model reliant on international journals and English as publication language. In the social sciences,

Journal of Data and the international journal model is dominant in Psychology and Social Health Information Science Sciences, whereas Economics, Educational Sciences, and Sociology show a 
Mapping Diversity of Publication Patterns in the Social Sciences and Humanities: An Approach Making Use of Fuzzy Cluster Analysis

Frederik T. Verleysen \& Arie Weeren

dispersed pattern across a broad spectrum of publication styles. Both Criminology and Political Sciences appear to be mostly similar to the humanities with a concentration of authors working in the national-journals-and-books model.

In general, any explanation of inter- and intra-disciplinary heterogeneity of publication patterns in the SSH should point to the intrinsic diversity of many aspects of scholarly research and information dissemination. Most humanities and social sciences are deeply fragmented with regard to intellectual interest and approach, conceptions of standards, as well as target audience (Hicks, 2004; Whitley, 2000). Specialization also relates to methodological differences, and these as well have an impact on the way in which scholarly work is published. In strongly quantitative fields of research, collaboration and ensuing co-authorship for the publication of journal articles is more easily achieved than in fields where qualitative methods are the norm (Kyvik, 2003; Moody, 2004).

Flemish sociologists, one of the cases documented in Section 3, can serve as a telling example of the way in which specialization can divide the researchers belonging to a single discipline. When clustered by means of the hard partitioning, the publication practices of sociologists show a distinctive pattern, with $47.7 \%$ belonging to Cluster One (international journals and English) and 52.3\% to Cluster Two (national journals and books) (Verleysen \& Weeren, 2016). Topical specialization does indeed explain this division to a considerable extent. A study from 2010 on publication patterns in Flemish Sociology has found that some communities of Flemish sociologists in more recent years have initiated an active participation in international communication networks (Vanderstraeten, 2010), which at the disciplinary level is attested to by growing shares of WoS-indexed journal articles (Engels et al., 2012) and English-language books published by prestigious international academic publishing houses (Verleysen \& Engels, 2014). In stark contrast, other research groups in Sociology in Flanders have retained a focus on studies at the national or regional level, with articles mainly in three Dutch-language journals published in Flanders or the Netherlands, which retain a strong national profile and hardly attract an international authorship or readership (Vanderstraeten, 2010).

Returning to the methodological point of view, the results of the fuzzy analysis presented in this paper are somewhat different from those of the hard partitioning previously conducted by Verleysen and Weeren (2016). Not only does the fuzzy result display additional information, it also avoids binary decisions for individual authors on cluster membership. This makes the result more complicated and slightly ambiguous. We note that especially for several humanities disciplines (Art History, History, Humanities General, Law, Literature and Theology) and two social sciences (Criminology and Political Sciences) the fuzzy result is indicative of gradual

Journal of Data and Information Science http://www.jdis.org 


\section{Research Paper}

differences between authors from the same discipline, a majority of which now lean towards the publication model in which national journals and books are the dominant publication types. This appears largely congruent with the traditional picture of research practices and information dissemination by humanities scholars. However, fuzzy cluster analysis of publication patterns at the author level does not result in equally less sharp internal divisions for all SSH disciplines. The cluster plots and probability density functions for four of the social sciences (Economics, Educational Sciences, Social Sciences General, and Sociology) point to a bifurcation of publication styles among researchers affiliated with the same discipline.

\section{Conclusion}

Cluster analysis has shown a valuable tool for the analysis of intra-disciplinary diversity of publication patterns in the social sciences and humanities. A fuzzy cluster analysis based on a prior hard partitioning results in a maximum of information: the partitioning based on the $k$-medoids algorithm allows for straightforward identification of the publication patterns underlying the clustering result, while the fuzzy principle shows for every individual author the degree of belonging to each cluster. In a final step, the shape of the probability density function shows for each discipline how publication styles are distributed over its authors and how heterogeneous scholarly fields of research really are.

All in all, this method for analyzing publication patterns seems well applicable to other bibliometric or research evaluation contexts, provided that the attributes of the cases to be clustered are derived from the actual scholarly research environment one wishes to analyze. The variables used for the Flemish case, or very similar ones, are probably also applicable to other non-Anglophone countries or regions (Verleysen \& Weeren, 2016).

\section{Acknowledgements}

The authors thank Truyken Ossenblok for making available the dataset used in this and a previous article, and its adaptation at our request. We also thank Tim Engels, Raf Guns, and Ronald Rousseau for their comments on a first manuscript of this paper. ECOOM is funded by the Flemish Government.

\section{Author Contributions}

The research questions were proposed by F.T. Verleysen (Frederik.Verleysen@uantwerpen.be, corresponding author). The data were collected by F.T. Verleysen (see also acknowledgements). The research framework was conceived by both authors. The programming in MATLAB ${ }^{\circledR} R 2016 \mathrm{a}$

Journal of Data and was carried out by A. Weeren (Arie.Weeren@mathworks.nl). F.T. Verleysen wrote and revised the final manuscript. 
Mapping Diversity of Publication Patterns in the Social Sciences and Humanities:

An Approach Making Use of Fuzzy Cluster Analysis

Frederik T. Verleysen \& Arie Weeren

\section{References}

Chi, P.S. (2015). Changing publication and citation patterns in political science in Germany. Scientometrics, 105, 1833-1848.

Dunn, J.C. (1976). Indices of partition fuzziness and the detection of clusters in large data sets. In M. Gupta (Ed.), Fuzzy Automata and Decision Processes. New York: Elsevier.

Engels, T.C.E., Ossenblok, T.L.B., \& Spruyt, E.H.J. (2012). Changing publication patterns in the social sciences and humanities, 2000-2009. Scientometrics, 93(2), 373-390.

Hicks, D. (2004). The four literatures of social science. In H. F. Moed, W. Glänzel, \& U. Schmoch (Eds.), Handbook of Quantitative Science and Technology Research: The Use of Publication and Patent Statistics in Studies of S\&T Systems (pp. 473-496). Dordrecht: Kluwer Academic.

Johnson, R.A., \& Wichern, D.W. (1992). Applied multivariate statistical analysis (Third ed.). New Jersey: Prentice Hall.

Kaufman, L., \& Rousseeuw, P.J. (1990). Finding groups in data: An introduction to cluster analysis. New York: Wiley.

Kyvik, S. (2003). Changing trends in publishing behaviour among university faculty, 1980-2000. Scientometrics, 58(1), 35-48.

Lin, Y., \& Kaid, L.L. (2000). Fragmentation of the intellectual structure of political communication study: Some empirical evidence. Scientometrics, 47(1), 143-164.

Moody, J. (2004). The structure of a social science collaboration network: Disciplinary cohesion from 1963-1999. American Sociological Review, 69(2), 213-239.

Nederhof, A.J. (2006). Bibliometric monitoring of research performance in the social sciences and the humanities: A review. Scientometrics, 66(1), 81-100.

Nederhof, A.J. (2011). A bibliometric study of productivity and impact of modern language and literature research. Research in Science Education, 20(2), 117-129.

Ossenblok, T.L.B. (2016). Scientific communication in the social sciences and humanities. Analysis of publication and collaboration in Flanders. Antwerp. (University of Antwerp Ph.D. dissertation)

Persson, O. (2015). Bibliometric analysis of two subdomains in philosophy: Free will and sorites. Scientometrics, 103(1), 47-73.

Puuska, H.M. (2014). Scholarly publishing patterns in Finland-a comparison of disciplinary groups. (doctoral dissertation), University ofTampere, Tampere, Finland.

Sivertsen, G. (2009). Publication patterns in all fields. In F. Aström, R. Danell, B. Larsen, \& J. W. Schneider (Eds.), Celebrating Scholarly Communication Studies: A Festschrift for Olle Persson at His $60^{\text {th }}$ Birthday (pp. 55-60). Leuven, Belgium: International Society for Scientometrics and Informetrics.

Vanderstraeten, R. (2010). Scientific communication: Sociology journals and publication practices. Sociology, 44, 559-576.

Verleysen, F.T., \& Weeren, A. (2016). Clustering by publication patterns of senior authors in the social sciences and humanities. Journal of Informetrics, 10, 254-272.

Whitley, R. (2000). The intellectual and social organization of the sciences. Oxford: Oxford University Press.

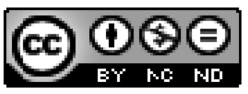

This is an open access article licensed under the Creative Commons-Attribution-Non CommercialNoDerivs License (http://creativecommons.org/licenses/by-nc-nd/4.0/).

Journal of Data and Information Science

http://www.jdis.org 


\section{Research Paper}

\section{Appendix ${ }^{\mathbb{1}}$}

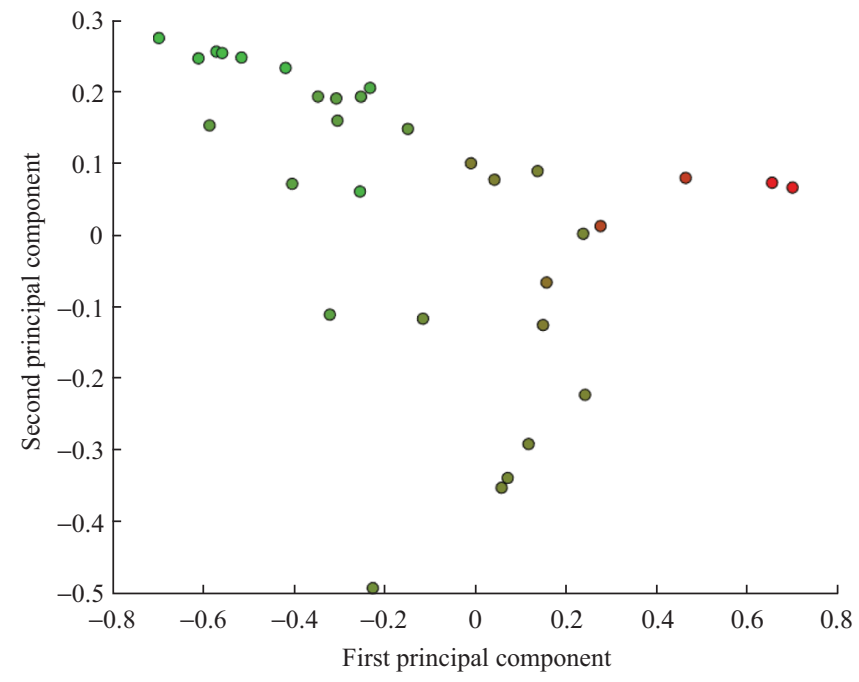

Figure 1. Fuzzy clustering of productive authors in Archeology $(n=32)$.

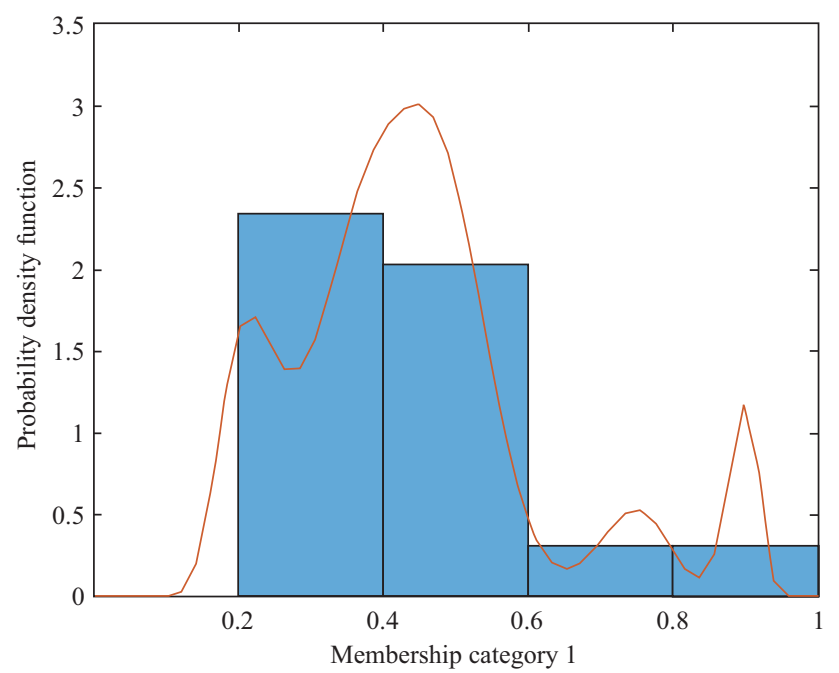

Figure 2. Probability density function of cluster membership coefficients for Archeology $(n=32)$. 
Mapping Diversity of Publication Patterns in the Social Sciences and Humanities: Frederik T. Verleysen \& Arie Weeren An Approach Making Use of Fuzzy Cluster Analysis

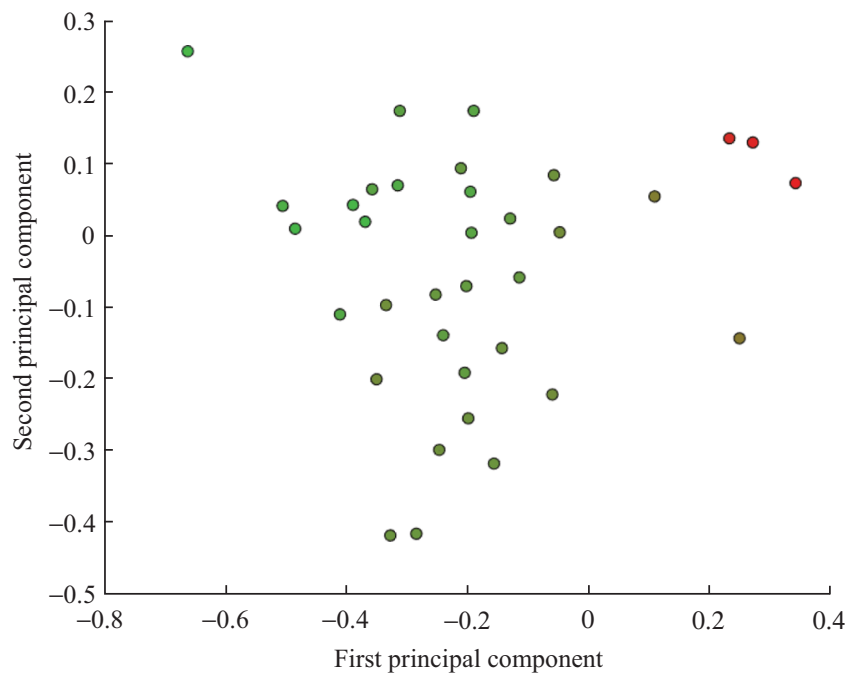

Figure 3. Fuzzy clustering of productive authors in Communication Studies $(n=35)$.



Figure 4. Probability density function of cluster membership coefficients for Communication Studies $(n=35)$. 


\section{Research Paper}

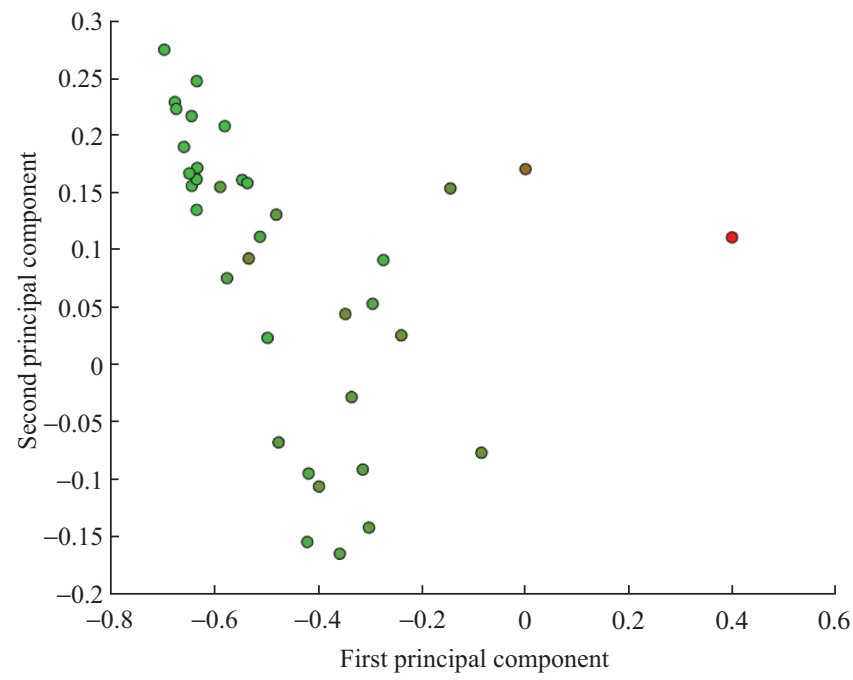

Figure 5. Fuzzy clustering of productive authors in Criminology $(n=39)$.

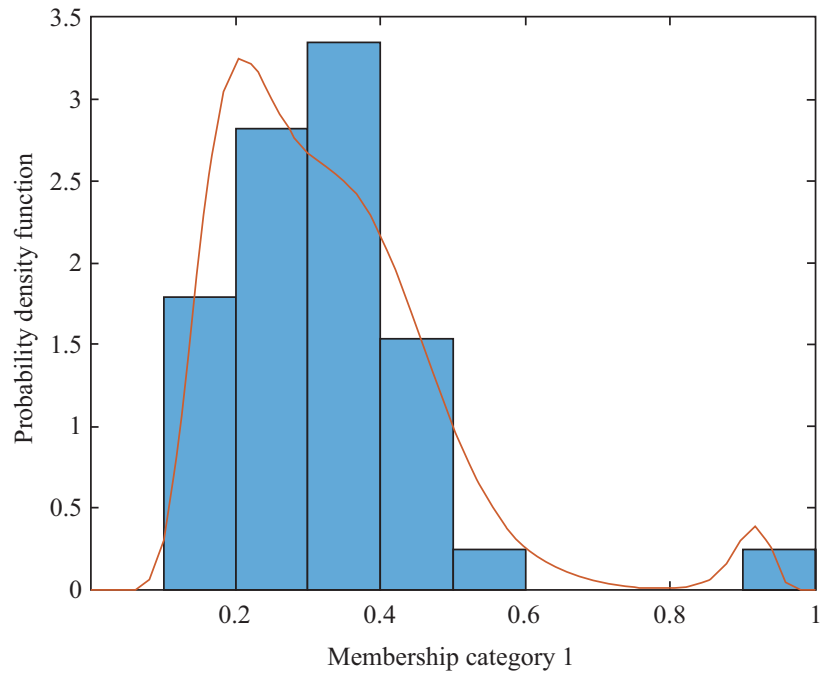

Figure 6. Probability density function of cluster membership coefficients for Criminology $(n=39)$. 


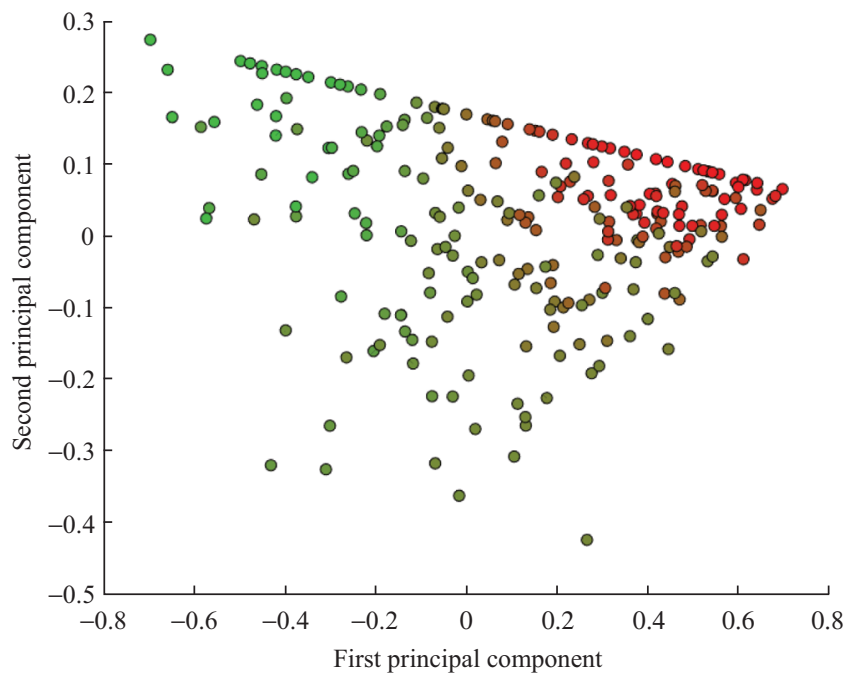

Figure 7. Fuzzy clustering of productive authors in Economics $(n=271)$.

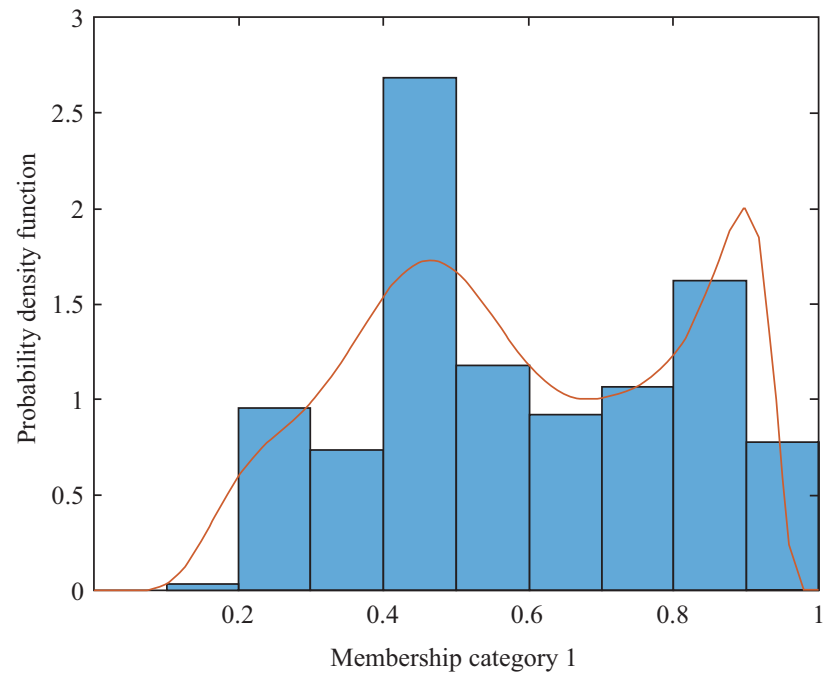

Figure 8. Probability density function of cluster membership coefficients for Economics $(n=271)$. 


\section{Research Paper}



Figure 9. Fuzzy clustering of productive authors in Educational Sciences $(n=96)$.



Figure 10. Probability density function of cluster membership coefficients for Educational Sciences $(n=96)$. 
Mapping Diversity of Publication Patterns in the Social Sciences and Humanities: Frederik T. Verleysen \& Arie Weeren An Approach Making Use of Fuzzy Cluster Analysis

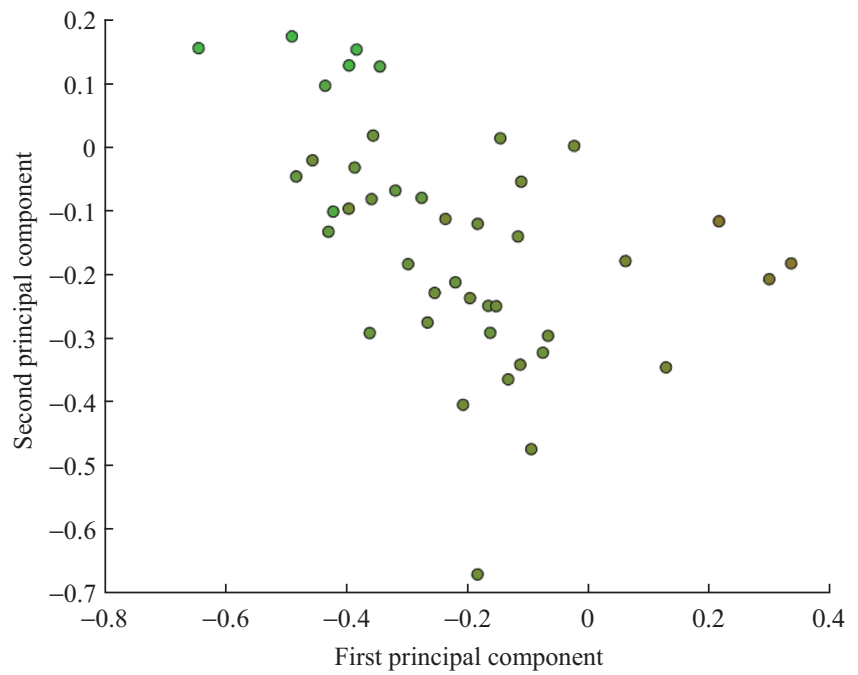

Figure 11. Fuzzy clustering of productive authors in Humanities General $(n=43)$.

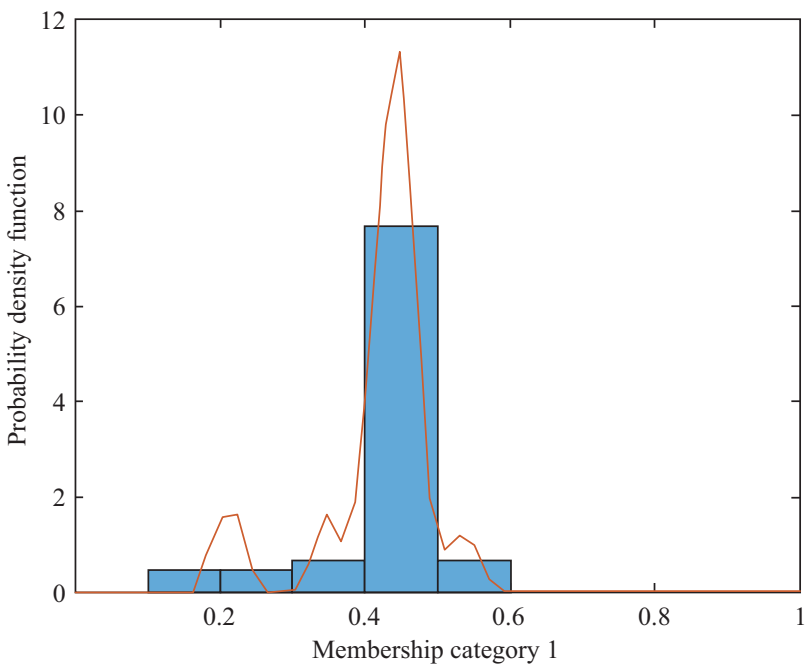

Figure 12. Probability density function of cluster membership coefficients for Humanities General $(n=43)$. 


\section{Research Paper}



Figure 13. Fuzzy clustering of productive authors in History $(n=66)$.

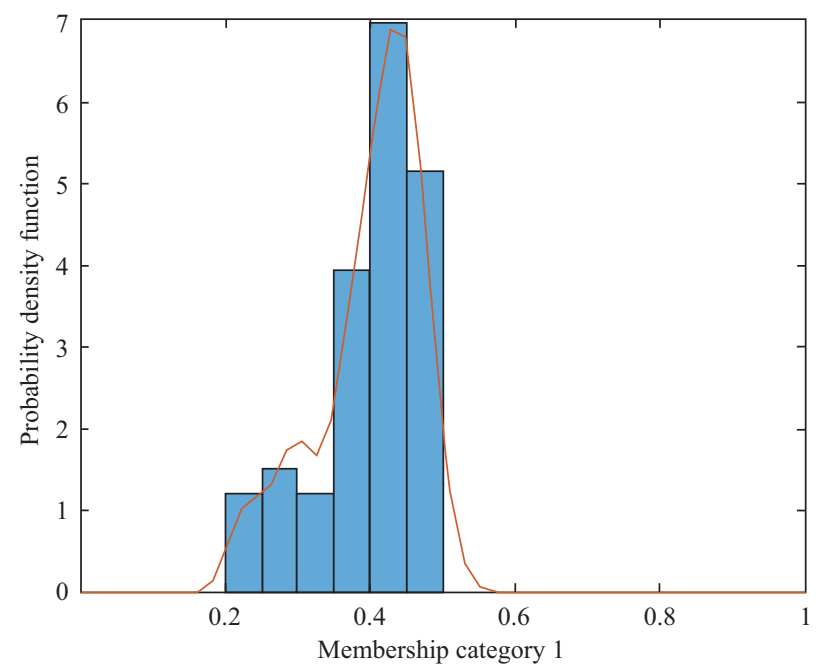

Figure 14. Probability density function of cluster membership coefficients for History $(n=66)$. 
Mapping Diversity of Publication Patterns in the Social Sciences and Humanities: An Approach Making Use of Fuzzy Cluster Analysis

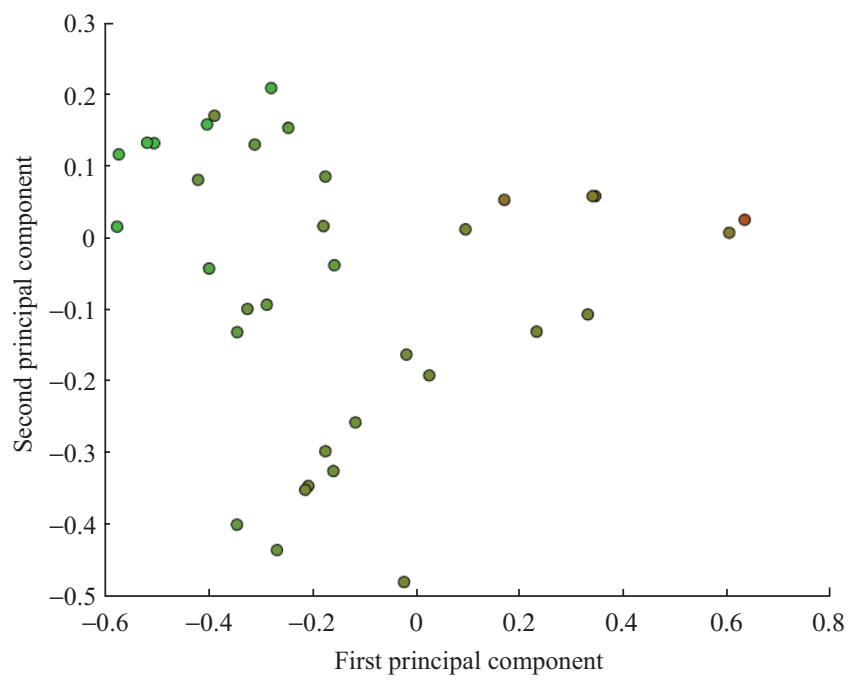

Figure 15. Fuzzy clustering of productive authors in History of Arts $(n=35)$.

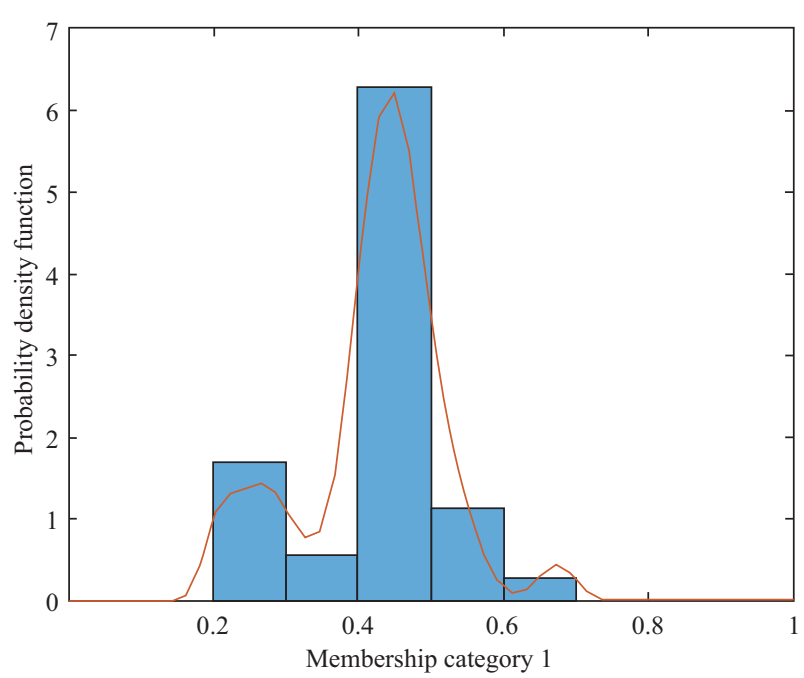

Figure 16. Probability density function of cluster membership coefficients for History of Arts $(n=35)$.
Frederik T. Verleysen \& Arie Weeren

Research Paper 


\section{Research Paper}

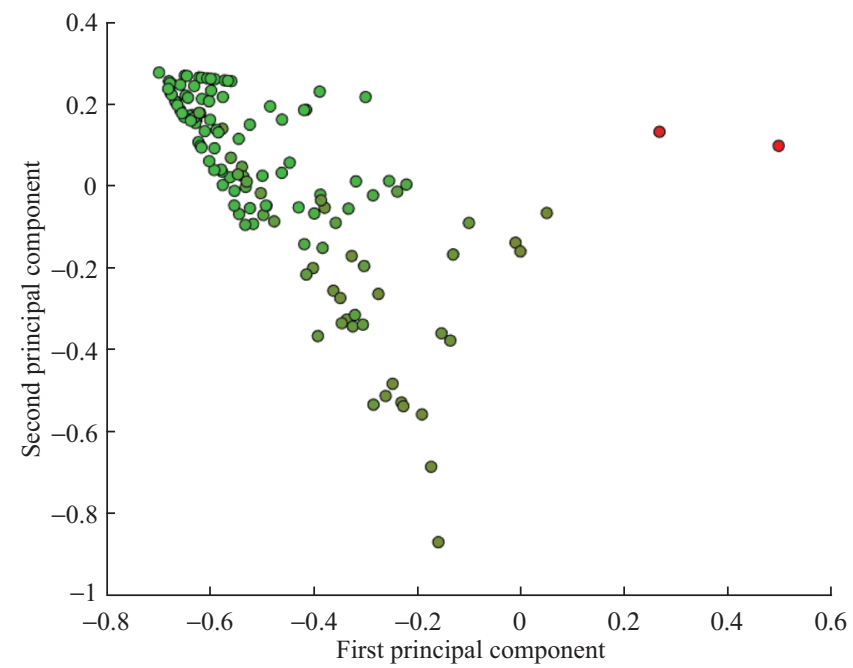

Figure 17. Fuzzy clustering of productive authors in Law $(n=206)$.

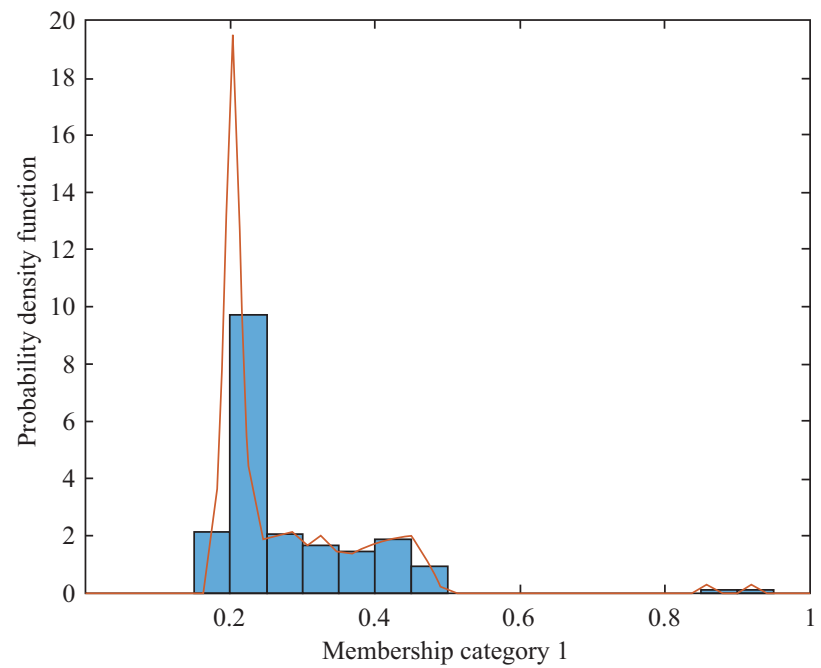

Figure 18. Probability density function of cluster membership coefficients for Law $(n=206)$. 
Mapping Diversity of Publication Patterns in the Social Sciences and Humanities: An Approach Making Use of Fuzzy Cluster Analysis

Frederik T. Verleysen \& Arie Weeren

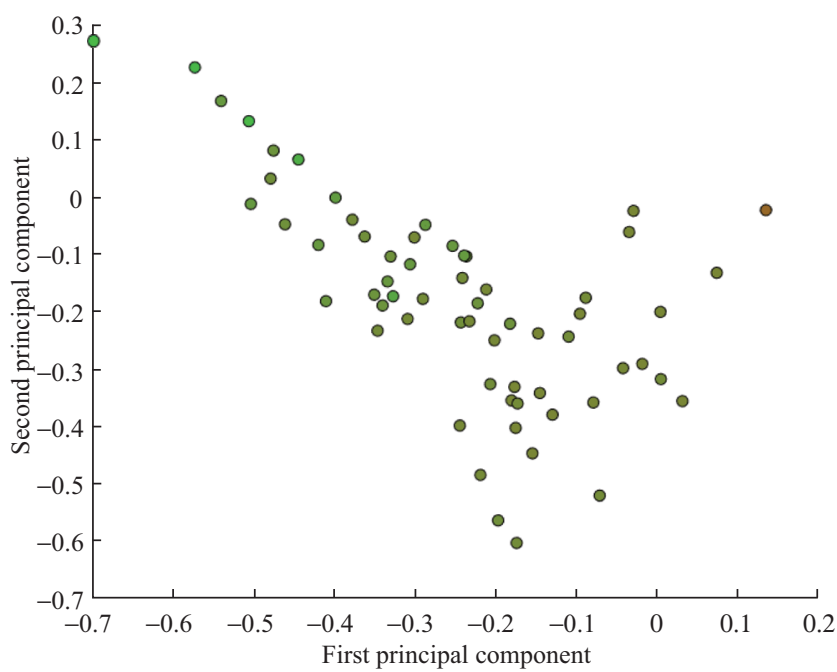

Figure 19. Fuzzy clustering of productive authors in Literature $(n=62)$.



Figure 20. Probability density function of cluster membership coefficients for Literature $(n=62)$. 


\section{Research Paper}



Figure 21. Fuzzy clustering of productive authors in Philosophy $(n=108)$.

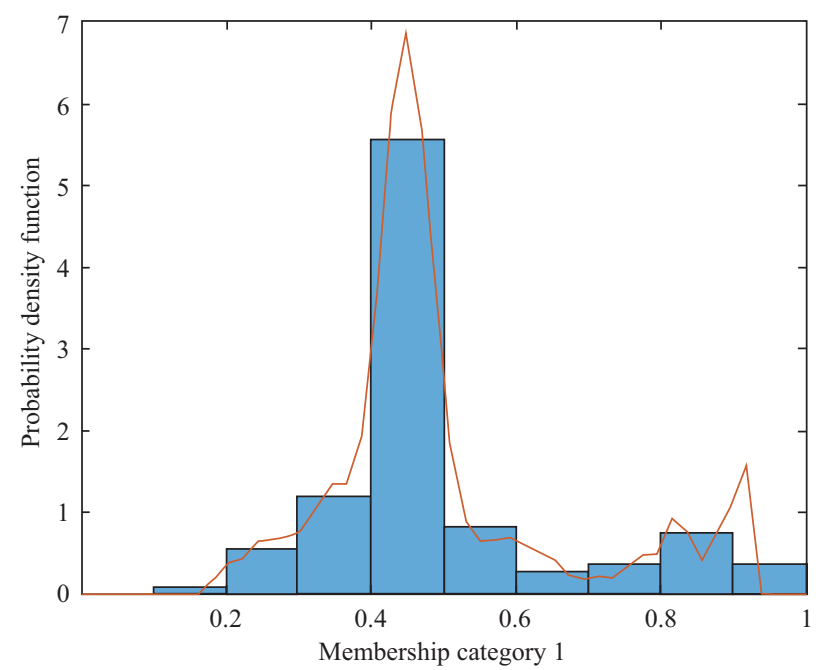

Figure 22. Probability density function of cluster membership coefficients for Philosophy ( $n=108)$. 
Mapping Diversity of Publication Patterns in the Social Sciences and Humanities: An Approach Making Use of Fuzzy Cluster Analysis

Frederik T. Verleysen \& Arie Weeren

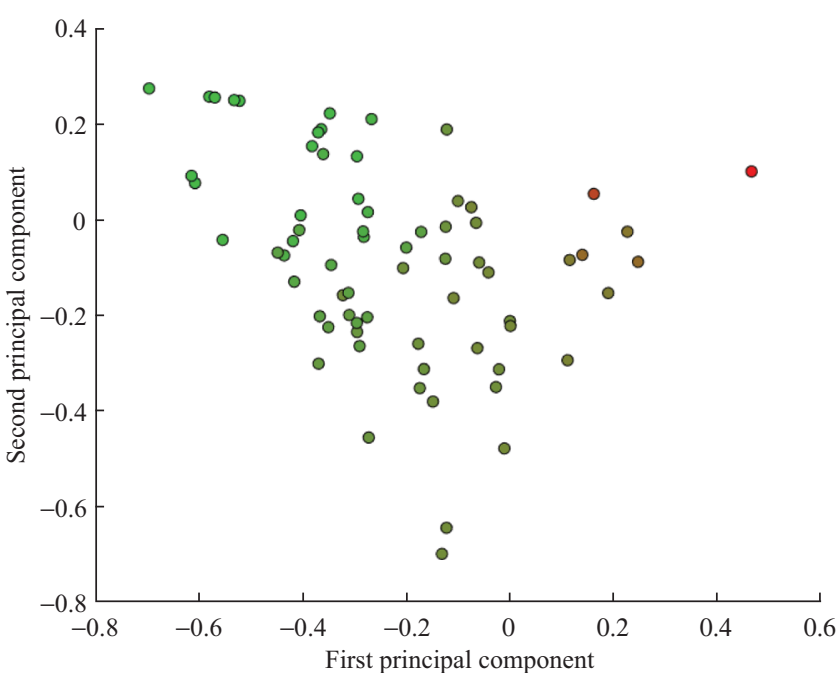

Figure 23. Fuzzy clustering of productive authors in Political Science $(n=74)$.



Figure 24. Probability density function of cluster membership coefficients for Political Science $(n=74)$. 


\section{Research Paper}

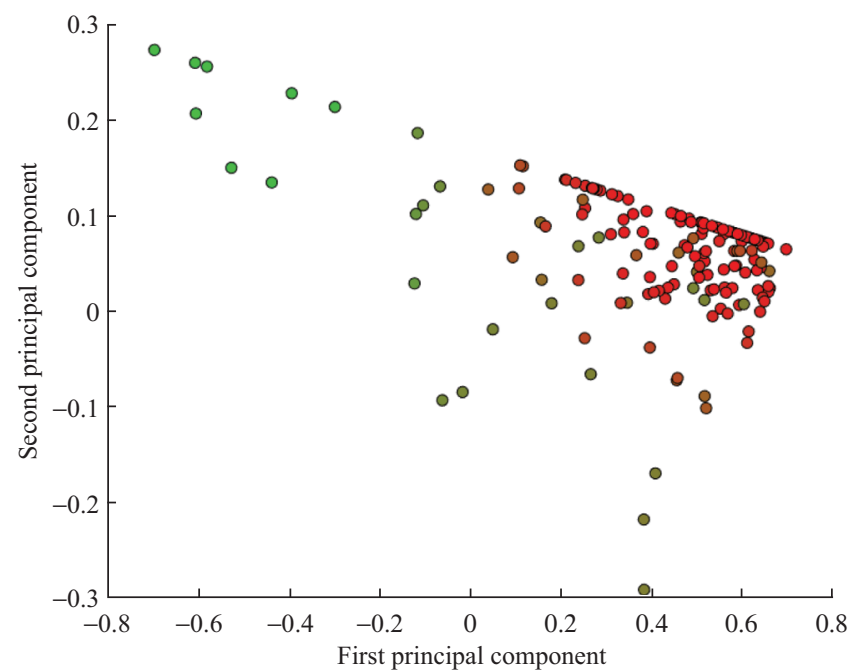

Figure 25. Fuzzy clustering of productive authors in Psychology $(n=199)$.



Figure 26. Probability density function of cluster membership coefficients for Psychology $(n=199)$. 
Mapping Diversity of Publication Patterns in the Social Sciences and Humanities: An Approach Making Use of Fuzzy Cluster Analysis

Frederik T. Verleysen \& Arie Weeren

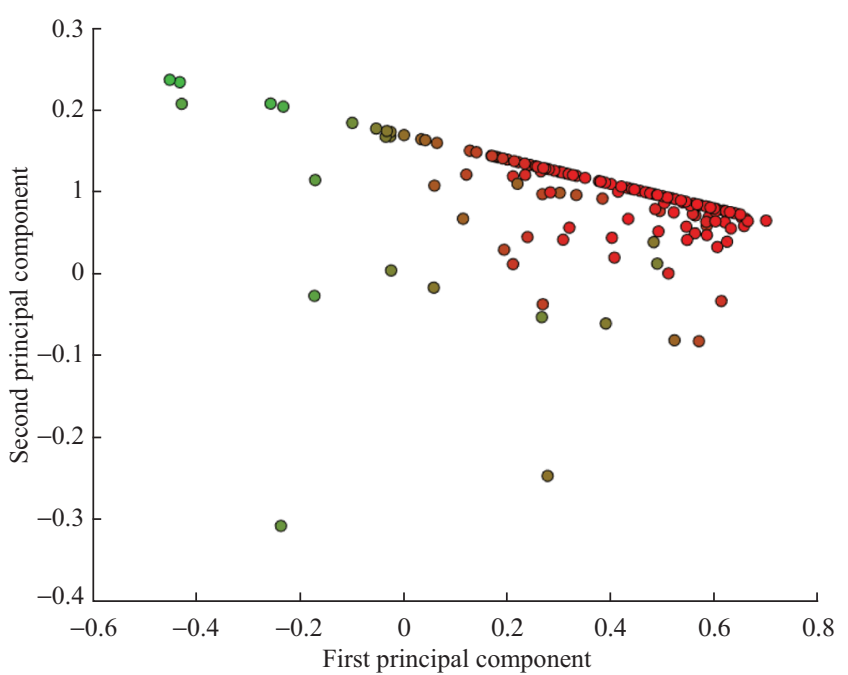

Figure 27. Fuzzy clustering of productive authors in Social Health Sciences $(n=228)$.

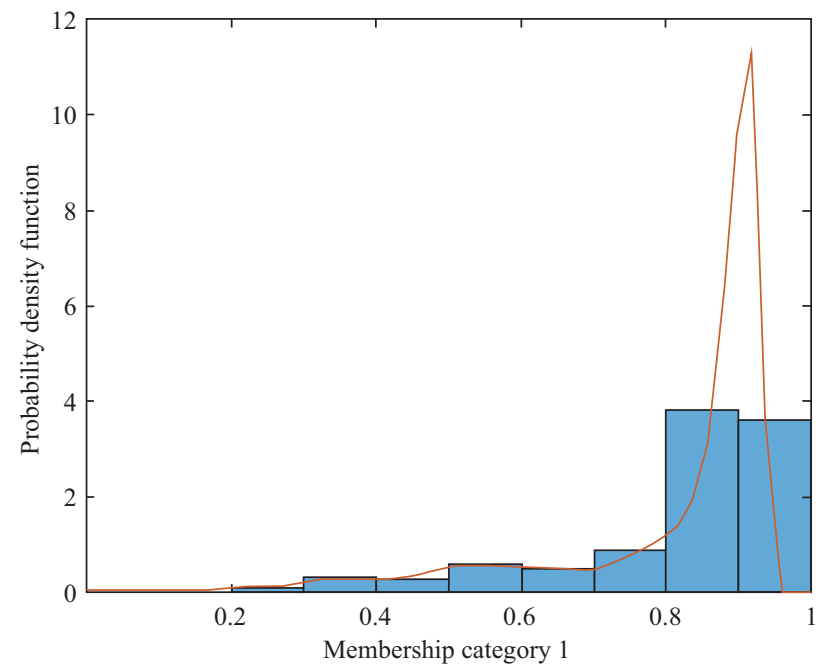

Figure 28. Probability density function of cluster membership coefficients for Social Health Sciences $(n=228)$. 


\section{Research Paper}

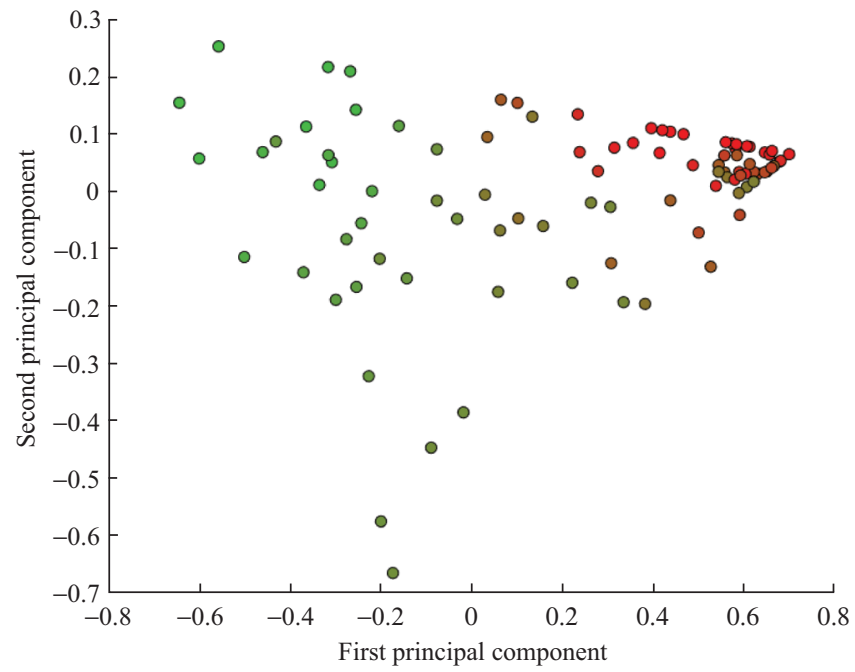

Figure 29. Fuzzy clustering of productive authors in Social Sciences General $(n=102)$.

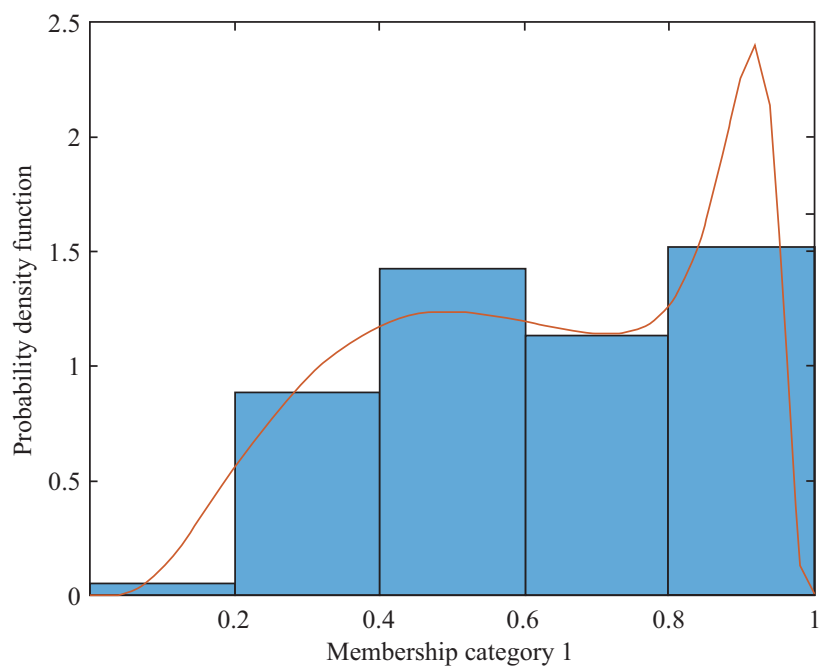

Figure 30. Probability density function of cluster membership coefficients for Social Sciences General $(n=102)$. 
Mapping Diversity of Publication Patterns in the Social Sciences and Humanities: An Approach Making Use of Fuzzy Cluster Analysis

Frederik T. Verleysen \& Arie Weeren

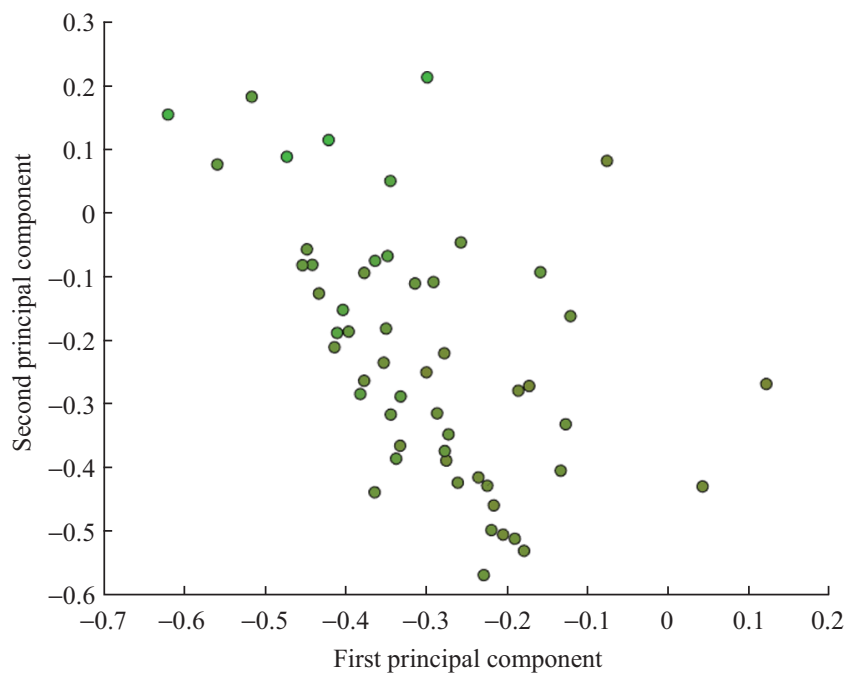

Figure 31. Fuzzy clustering of productive authors in Theology $(n=54)$.



Figure 32. Probability density function of cluster membership coefficients for Theology ( $n=54)$. 